\title{
Risk Factors of a Pulmonary Thromboembolism After Colorectal Surgery
}

\author{
Junyub Kim, Byung-Noe Bae, Hyun Seok Jung, Inseok Park, Hyunjin Cho, Geumhee Gwak, Kiwhan Kim, \\ Hong-Joo Kim, Young Duk Kim \\ Department of Surgery, Inje University Sanggye Paik Hospital, Seoul, Korea
}

Purpose: Previous studies have revealed that predictors and risk factors of pulmonary thromboembolism (PTE) are malignancy, immobilization, diabetes, and obesity in the postoperative patients. However, in patients undergoing colorectal cancer, studies of PTE have not been enough. Thus, we investigated the risk factors of PTE related to colorectal surgery.

Methods: From January 2009 to October 2014, 312 patients received colorectal surgery without other organ resection. The postoperative patients with PTE were 14 (4.5\%), and they were classified by sex, age, and stage as a 1:3 paired match to the control group. A multiple logistic regression was performed to identify which factors were associated with PTE.

Results: One patient was in stage I, 3 in stage II, 9 in stage III, and 1 in stage IV. In the binary logistic regression analysis, history of diabetes mellitus (odds ratio, 6.498; $\mathrm{P}=0.031$ ) and being overweight (odds ratio, 10.018; $\mathrm{P}=0.014$ ) were independent risk factors for PTE in patients undergoing colorectal cancer.

Conclusion: A history of diabetes mellitus and being overweight were independent risk factors of PTE after colorectal cancer.

Keywords: Colorectal neoplasms; Pulmonary embolism; Antineoplastic agents

\section{INTRODUCTION}

A venous thromboembolism (VTE) may occur following a surgical procedure for a malignant neoplasm. The incidence of VTE after colorectal cancer surgery ranges from $1.4 \%$ to $2.4 \%$ [1-3]. VTE is one of the leading causes of significant morbidity and mortality in cancer patients after surgery. In particular, a pulmonary thromboembolism (PTE) is associated with higher rates of mortality compared with thromboembolisms found in other organs [1]. Even when a PTE occurs, early detection and management of the PTE can avert unfavorable consequences in many cancer patients. Clinicians need to be vigilant on the selection of

Received: August 27, 2015 - Accepted: October 3, 2015

Correspondence to: Byung-Noe Bae, M.D.

Department of Surgery, Inje University Sanggye Paik Hospital, 1342

Dongil-ro, Nowon-gu, Seoul 01757, Korea

Tel: +82-2-950-1021, Fax: +82-2-3391-4393

E-mail: bnbae@paik.ac.kr

(c) 2015 The Korean Society of Coloproctology

This is an open-access article distributed under the terms of the Creative Commons Attribution NonCommercial License (http://creativecommons.org/licenses/by-nc/3.0) which permits unrestricted noncommercial use, distribution, and reproduction in any medium, provided the original work is properly cited. cancer patients with risk factors of PTE before and after surgery, with more careful monitoring in routine follow-up, and this strategy appears to be important in achieving good results. In addition, prophylactic anticoagulation therapy may be considered for cancer patients classified as a high-risk group for PTE before surgery $[4,5]$. Thus, the monitoring and evaluation of preoperative PTE-related risk factors is of great importance in reducing morbidity and mortality after colorectal cancer surgery. A number of studies related to the usefulness of prophylactic anticoagulation therapy before surgery and risk factors for VTE have been conducted thus far [6-9]. Prophylactic anticoagulation therapy with low-molecular-weight heparin (LMWH) may postoperatively reduce the incidence of VTE in patients with risk factors of VTE [10]. Risk factors of VTE are malignant tumor, older age, the level of physical activity, diabetes mellitus, obesity, and heart disease [11]. According to an evidence-based clinical practice guideline of the American Society of Clinical Oncology, prophylaxis and treatment of VTE-associated risk factors is recommended in patients with malignancy. In VTE patients, PTE had a higher motality. In colorectal cancer, PTE incidence was 4.1 folds higher than other malignacy [12]. With regard to the development of PTE associated with postoperative colorectal cancer surgery $[11,13]$, local studies 
have been less robust in Korea.

At present, early diagnosis of colorectal cancer and rapid technical advancements have resulted in a gradual increase in the survival rate for colorectal cancer patients. Minimizing the complications during the treatment and the follow-up periods is a very critical issue in improving the quality of life for people with colorectal cancer. In consideration of this, the authors attempted to identify clinicopathologic factors influencing the development of PTE in patients with colorectal cancer, with an aim to find possible methods to reduce the incidence of PTE-associated morbidity and mortality.

\section{METHODS}

We evaluated 414 patients who had undergone colorectal cancer surgery between January 2009 and October 2014 at the Department of Surgery, Inje University Sanggye Paik Hospital, via a retrospective review of their medical records. Institutional Review Board approval was obtained from Inje University Sanggye Paik Hospital (IRB No. 2015-05-18). After patients with a history of synchronous organ resection at other metastatic sites and deep vein thrombosis (DVT) had been excluded, a total of 312 patients with colorectal cancer were included for our retrospective analysis. PTE was postoperatively diagnosed by using chest computed tomography (CT) before initiation of chemotherapy or regular clinical laboratory tests. Patients underwent routine chest and abdominal CT scans every 3 months after the surgical procedure for the first year, then every 6 months for 2 years, and every year thereafter. For recurrent colorectal cancer, chest CT scans were performed prior to chemotherapy. Of the 312 patients screened, a total of 14 patients (4.5\%) were finally diagnosed with PTE, and these patients underwent a chest CT scan every 3 months (Fig. 1).

To reduce patient selection bias, we compared 14 PTE patients with 42 control subjects without PTE in a sex-, age- and tumorstage-matched ratio (1:3). Then, clinicopathologic factors affect-

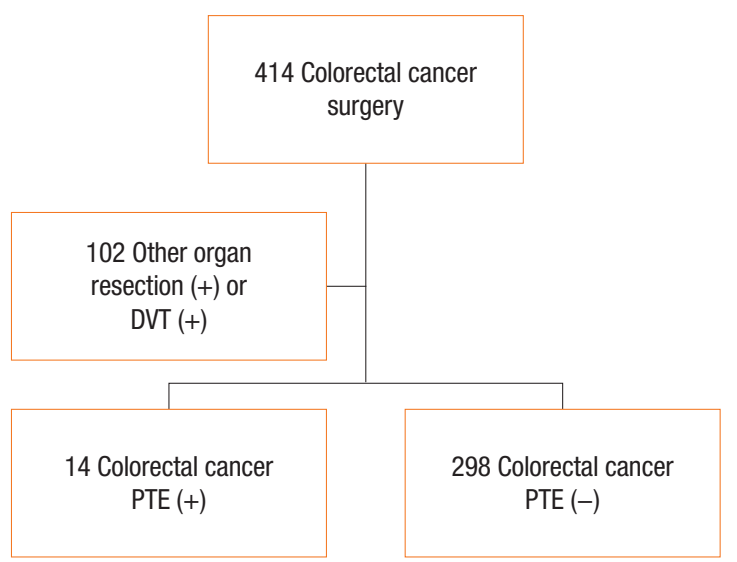

Fig. 1. Flow chart of the study. PTE, pulmonary thromboembolism; DVT, deep vein thrombosis. ing the development of PTE were compared between the two groups. The clinicopathologic factors assessed were a history of diabetes mellitus and hypertension, body mass index (BMI), overweight according to the Asia-Pacific guidelines of obesity classification, smoking, and chest pain/dyspnea during follow-up. The preoperative evaluation factors also included the American Society of Anesthesiologist (ASA) score and the route of administration in postoperative chemotherapy. In addition, blood parameters, including blood pathologies, such as complete blood counts, prothrombin time (PT), partial thromboplastin time, PT international normalized ratio, liver function test, and albumin, total protein, sodium, potassium, chloride, and cholesterol levels, were compared. All statistical analyses were performed using MedCalc statistical software ver. 15.5 (MedCalc Software bvba, Ostend, Belgium; https://www.medcalc.org; 2015). In the presence of nonnormal distribution data, analyses were performed by using the Mann-Whitney $\mathrm{U}$ test for continuous data and the chi-square test for categorical data. For the independent PTE risk factor analysis, a multivariate logistic regression analysis was done with statistically significant variables. A P-value of $<0.05$ was considered statistically significant.

\section{RESULTS}

Of the 312 patients included in this study, we finally diagnosed 14 patients with PTE (PTE group) and compared them with 42 control subjects without PTE (control group) according to a tumorstage-, age-, and sex-matched ratio (1:3). The mean age was 69 years in both groups. Eight males and 6 females were in the PTE group. The distribution of clinical stages of colorectal cancer in the PTE group was as follows: stage I (1 patient), stage II (4 patients), stage III (9 patients), and stage IV (1 patient). The diagnosis interval for PTE was from 1 to 47 months, and the median diagnosis interval was 14 months. PTE patients were followed up every 3 months by using chest CT scans, and prophylactic anticoagulation therapy with LMWH was performed. PTE-associated symptoms were not observed in all PTE patients during followup; neither were death nor any signs of exacerbation.

The number of PTE patients aged 70 or older was 7 compared with 21 in the control group, but the difference was not significant. Preoperative diabetes mellitus showed a higher proportion in the PTE group than in the control group (6 PTE patients vs. 7 control subjects before surgery, $\mathrm{P}=0.006$ ). Seven PTE patients and 25 control subjects were diagnosed with preoperative hypertension, but the difference was insignificant. Routine monitoring did not reveal any differences in the incidences of dyspnea and chest discomfort between the two groups after the surgical procedure. The ASA scores were compared in the PTE group and the control group, respectively: ASA 1 (none vs. 9 patients), ASA 2 (12 patients vs. 24 patients), ASA 3 (2 patients vs. 8 patients), and ASA 4 (none vs. 1 patient). No significant differences in the ASA scores between the two groups were observed, not even for an 
Table 1. Medical history of patients

\begin{tabular}{lccc}
\hline Variable & PTE $(+)(n=14)$ & PTE $(-)(n=42)$ & P-value \\
\hline Age $(>70 \mathrm{yr})$ & 7 & 21 & 1.000 \\
Diabetes mellitus & 7 & 6 & 0.006 \\
Hypertension & 7 & 25 & 0.533 \\
Deep vein thrombosis & 0 & 0 & - \\
ASA score $(\geq 3)$ & 2 & 9 & 0.560 \\
Overweight $\left(\mathrm{BMl}>23 \mathrm{~kg} / \mathrm{m}^{2}\right)$ & 10 & 11 & 0.002 \\
Intravenous chemotherapy & 10 & 23 & 0.209 \\
Dyspnea & 0 & 1 & 0.560 \\
Chest pain & 0 & 2 & 0.406 \\
History of smoking & 2 & 4 & 0.618 \\
Transfusion & 0 & 3 & 0.304 \\
\hline
\end{tabular}

PTE, pulmonary thromboembolism; ASA, American Society of Anesthesiologists; BMI, body mass index.

Table 3. Logistic regression of risk factors of PTE after colorectal surgery

\begin{tabular}{lrcc}
\hline Variable & Odds ratio & $\begin{array}{c}95 \% \text { Confidence } \\
\text { interval }\end{array}$ & P-value \\
\hline Male sex & 3.196 & $0.543-18.814$ & 0.199 \\
Age $(>70 \mathrm{yr})$ & 2.052 & $0.406-10.373$ & 0.385 \\
Overweight $\left(\mathrm{BMl}>23 \mathrm{~kg} / \mathrm{m}^{2}\right)$ & 10.018 & $1.596-62.872$ & 0.014 \\
Diabetes mellitus & 6.498 & $1.189-35.506$ & 0.031 \\
ASA score $(\geq 3)$ & 0.213 & $0.019-2.392$ & 0.210 \\
\hline
\end{tabular}

PTE, pulmonary thromboembolism; BMI, body mass index; ASA, American Society of Anesthesiologists.

ASA score $>3$. Although the mean BMI value did not show a statistical difference between the two groups (PTE (+) $24.4 \mathrm{~kg} / \mathrm{m}^{2}$ vs. PTE (-) $22.75 \mathrm{~kg} / \mathrm{m}^{2}, \mathrm{P}=0.119$ ), a statistical significance was observed for being overweight according to the Asia-Pacific obesity classification (10 PTE patients vs. 11 control subjects, $\mathrm{P}=0.002$ ). Except for three individuals who did not receive chemotherapy due to systemic weakness (one PTE patient vs. two control subjects [including clinical stage I]), all patients underwent chemotherapy treatment. Intravenous chemotherapy was performed in 10 PTE patients and 23 control subjects, respectively, but no differences between the two groups were observed. With regard to the chemotherapeutic agents, oral therapy consisted of UFTE (tegafur, uracil) and doxifluridine while intravenous regimens included FOLFOX (folinic acid + 5-fluorouracil + oxaliplatin), FOLFIRI (folinic acid + 5-fluorouracil + irinotecan), and XELOX (oxaliplatin + capecitabine) (Table 1). No differences in blood pathology findings between the two groups were noted (Table 2).

A multivariate logistic regression analysis of additional variables, including sex, older age (71 years or older), and ASA score (more than ASA 3) was performed based on previous studies and in-
Table 2. Laboratory results of patients

\begin{tabular}{|c|c|c|c|}
\hline Variable & PTE $(+)(n=14)$ & $\operatorname{PTE}(-)(n=42)$ & P-value \\
\hline PT (\%) & $86.7 \pm 18.06$ & $86.8 \pm 13.58$ & NS \\
\hline aPTT (sec) & $25.4 \pm 3.64$ & $28.6 \pm 7.70$ & NS \\
\hline INR & $1.1 \pm 0.10$ & $1.1 \pm 0.26$ & NS \\
\hline Cholesterol (mg/dL) & $124.5 \pm 64.13$ & $155.0 \pm 51.25$ & NS \\
\hline Sodium (mEq/L) & $138.0 \pm 2.88$ & $139.1 \pm 2.24$ & NS \\
\hline Potassium (mEq/L) & $4.3 \pm 0.40$ & $4.0 \pm 0.47$ & NS \\
\hline Chloride (mEq/L) & $102.1 \pm 3.22$ & $104.8 \pm 3.52$ & NS \\
\hline Hemoglobin (d/dL) & $12.2 \pm 2.01$ & $11.8 \pm 2.05$ & NS \\
\hline Hematocrit (\%) & $37.4 \pm 5.31$ & $35.6 \pm 5.23$ & NS \\
\hline WBC $\left(\times 10^{3} / \mu \mathrm{L}\right)$ & $7.2 \pm 2.88$ & $7.0 \pm 2.39$ & NS \\
\hline Platelet ( $\mu \mathrm{L})$ & $290.7 \pm 125.97$ & $281.3 \pm 107.58$ & NS \\
\hline Albumin (g/dL) & $3.7 \pm 0.96$ & $3.7 \pm 0.63$ & NS \\
\hline Total protein (g/dL) & $6.1 \pm 1.44$ & $6.6 \pm 0.80$ & NS \\
\hline AST (IU) & $31.3 \pm 38.69$ & $24.4 \pm 11.07$ & NS \\
\hline ALT (IU) & $19.1 \pm 12.63$ & $14.5 \pm 8.78$ & NS \\
\hline ALP (U/L) & $69.9 \pm 21.59$ & $80.3 \pm 24.75$ & NS \\
\hline
\end{tabular}

Values are presented as mean \pm standard deviation.

PTE, pulmonary thromboembolism; PT, prothrombin time; aPTT, activated partial thromboplastin time; INR, international normalized ratio; WBC, white blood cell; AST, aspartate aminotransferase; ALT, alanine aminotransferase; ALP, alkaline phosphatase; NS, nonspecific.

cluded a history of being overweight and having diabetes mellitus, which were significant factors in the univariate analysis. Results suggested that independent risk factors were a history of diabetes mellitus (odds ratio, 6.498; $\mathrm{P}=0.031$ ) and being overweight (odds ratio, 10.018; $\mathrm{P}=0.014$ ) whereas other risk factors did not show any statistical significance (Table 3 ).

\section{DISCUSSION}

A large-scale, nationwide, retrospective study, which was designed to investigate a link between colorectal surgery and the development of VTE and PTE in the United States, found that VTE or PTE was clinically recognized in $2 \%$ of patients, with a close relationship with the following risk factors: malignant neoplasm, laparotomy, hypoalbuminemia, steroid treatment, obesity, older age, emergency admission, and ASA score (more than ASA 2) [14]. Risk factors associated with DVT and PTE in cancer patients were reported in a number of studies in Western countries, where multiple risk factors, such as diabetes mellitus, level of physical activity in patients, cardiac disorder and ethnicity, were presented $[1-3,15]$. Although a local study related to the development of postoperative VTE in Korean cancer patients showed that the incidence of VTE in patients who underwent colorectal cancer surgery was higher than it was in patients who underwent other types of surgeries, its occurrence in the Korean population was 
lower than that in the Western population [16]. In other local studies on the incidence of postoperative VTE in patients with colorectal cancer, a lower survival rate was reported, but the fact that VTE was an independent risk factor that lowered the survival rate was not delineated $[17,18]$. Overall, these studies did not identify any risk factors associated with VTE and PTE.

The number of PTE patients in our study, which was $4.5 \%$ of the 312 patients screened, was somewhat higher than that reported in local studies. We postulated that the higher proportion of PTE patients might be attributable to the nonuse of prophylactic anticoagulation therapy before surgery in our clinic. Now that the Westernized physique and constitution of people in Korea and Asia have become visible, prophylactic anticoagulation therapy should be strongly considered in patients with risk factors for PTE. PTE is generally diagnosed via chest CT angiography, but in this study, PTE was accidently found during routine examination, with no diagnostic tests to address any acute symptoms. Tresoldi et al. [19] reported that routine chest CT was more advantageous than diagnostic tests in cancer patients, considering the small risk of patient exposure to radiation from CT after intravenous contrast injection.

The level of physical activity in cancer patients has been well known to be one of the risk factors for PTE [15]. Earlier studies of cancer patients indicated that the ASA score was higher in the PTE group than in the non-PTE group [14]. However, the results of our study did not reveal the ASA score to be one of the significant risk factors for the development of PTE. Based on other prior findings, we deem that the level of physical activity should be studied in a large population, coupled with additional analyses of other risk factors, including hospitalization period and surgery time, as well as ASA score.

A malignant neoplasm has been shown to be one of the risk factors for DVT and PTE [11]. The chemotherapeutic agent is a factor that increases thrombus risk in cancer patients [15]. In this study, no differences in the chemotherapy-associated incidence of PTE were observed. Because an investigation of the effects of antitumor drugs on the development of PTE as a risk factor may appear to have many limitations, research focusing on adverse reactions to drugs administered following colorectal cancer surgery would be helpful.

In our study, a higher proportion of patients with a history of diabetes mellitus before surgery were significantly observed in the PTE group ( $\mathrm{P}=0.006)$; thus, it can be considered to be an independent risk factor (odds ratio, 6.498; $\mathrm{P}=0.031$ ). Previous findings indicated that diabetes mellitus affected the incidence of PTE preoperatively and postoperatively [20]. Like other reports, this study also showed that diabetes mellitus may have contributed to PTE risk as a preceding factor. Numerous prior studies identified that high BMI increased the incidence of postoperative PTE in patients with colorectal cancer [15]. In the present study, we observed that most PTE patients were obese based on the Asia-Pacific obesity classification, which is in agreement with previous findings. As previously reported in many studies, diabetes mellitus and being overweight are risk factors for PTE, and the connection was statistically significant in our study. Because of prior awareness that diabetes mellitus or obesity is associated with increased risk of PTE, clinicians should establish more aggressive prevention and treatment strategies for cancer patients with such risk factors before and after surgery. With regard to the two risk factors for PTE mentioned in our study, a long-term study will be necessary if the incidence of PTE is to be reduced via proper treatment. In addition, both diabetes mellitus and obesity are related to metabolic syndrome, so relevant factors should be further studied.

In the present study, we diagnosed postoperative PTE through routine observation for treatment whereas PTE-related symptoms or death was not observed. We also found that routine follow-up tests using chest CT scans were significantly meaningful in detecting and treating PTE in patients undergoing colorectal cancer surgery and chemotherapy. However, if the risk factors of postoperative PTE are taken into account, clinicians should establish a thorough follow-up program to ensure more timely tests and treatment in a high-risk group.

The results of our study showed that both diabetes mellitus and being overweight were risk factors for postoperative PTE in patients with colorectal cancer. However, we were limited to analyzing intrapatient characteristics due to the small sample size. Further studies are needed to identify other risk factors, such as medical condition that reduces the level of physical activity, histopathological features, and comorbid colorectal disease. Multicenter and comparative studies for risk factors of PTE are also necessary. Through such research, the risk factors of PTE may be thoroughly studied for more effective prevention and treatment of PTE.

In summary, our results suggest that diabetes mellitus and overweight are risk factors for postoperative PTE in patients with colorectal cancer. In this context, clinicians should be vigilant in closely investigating the medical history, the level of physical activity and the BMI before surgery in these patients. Then, they should identify risk factors of postoperative PTE during followup to ensure proper individual management. Further studies should clarify the associations between these risk factors for colorectal cancer and the development of PTE.

\section{CONFLICT OF INTEREST}

No potential conflict of interest relevant to this article was reported.

\section{REFERENCES}

1. Anderson FA Jr, Wheeler HB, Goldberg RJ, Hosmer DW, Patwardhan NA, Jovanovic B, et al. A population-based perspective of the hospital incidence and case-fatality rates of deep vein thrombosis and pulmonary embolism. The Worcester DVT Study. Arch In- 
tern Med 1991;151:933-8.

2. Duff J, Walker K, Omari A, Stratton C. Prevention of venous thromboembolism in hospitalized patients: analysis of reduced cost and improved clinical outcomes. J Vasc Nurs 2013;31:9-14.

3. Shapiro R, Vogel JD, Kiran RP. Risk of postoperative venous thromboembolism after laparoscopic and open colorectal surgery: an additional benefit of the minimally invasive approach? Dis Colon Rectum 2011;54:1496-502.

4. Horlander KT, Mannino DM, Leeper KV. Pulmonary embolism mortality in the United States, 1979-1998: an analysis using multiple-cause mortality data. Arch Intern Med 2003;163:1711-7.

5. Connors JM. Prophylaxis against venous thromboembolism in ambulatory patients with cancer. N Engl J Med 2014;370:2515-9.

6. Blom JW, Doggen CJ, Osanto S, Rosendaal FR. Malignancies, prothrombotic mutations, and the risk of venous thrombosis. JAMA 2005;293:715-22.

7. Chew HK, Wun T, Harvey D, Zhou H, White RH. Incidence of venous thromboembolism and its effect on survival among patients with common cancers. Arch Intern Med 2006;166:458-64.

8. Alcalay A, Wun T, Khatri V, Chew HK, Harvey D, Zhou H, et al. Venous thromboembolism in patients with colorectal cancer: incidence and effect on survival. J Clin Oncol 2006;24:1112-8.

9. Cronin-Fenton DP, Sondergaard F, Pedersen LA, Fryzek JP, Cetin $\mathrm{K}$, Acquavella J, et al. Hospitalisation for venous thromboembolism in cancer patients and the general population: a populationbased cohort study in Denmark, 1997-2006. Br J Cancer 2010;103: 947-53.

10. Lyman GH, Khorana AA, Kuderer NM, Lee AY, Arcelus JI, Balaban EP, et al. Venous thromboembolism prophylaxis and treatment in patients with cancer: American Society of Clinical Oncology clinical practice guideline update. J Clin Oncol 2013;31:2189-204.

11. McNally MP, Burns CJ. Venous thromboembolic disease in colorectal patients. Clin Colon Rectal Surg 2009;22:34-40.

12. Huber O, Bounameaux H, Borst F, Rohner A. Postoperative pulmonary embolism after hospital discharge: an underestimated risk. Arch Surg 1992;127:310-3.

13. Lyman GH, Khorana AA, Falanga A, Clarke-Pearson D, Flowers C, Jahanzeb M, et al. American Society of Clinical Oncology guideline: recommendations for venous thromboembolism prophylaxis and treatment in patients with cancer. J Clin Oncol 2007;25:5490505.

14. Moghadamyeghaneh Z, Hanna MH, Carmichael JC, Nguyen NT, Stamos MJ. A nationwide analysis of postoperative deep vein thrombosis and pulmonary embolism in colon and rectal surgery. J Gastrointest Surg 2014;18:2169-77.

15. Otten HM, Mathijssen J, ten Cate H, Soesan M, Inghels M, Richel DJ, et al. Symptomatic venous thromboembolism in cancer patients treated with chemotherapy: an underestimated phenomenon. Arch Intern Med 2004;164:190-4.

16. Yhim HY, Jang MJ, Bang SM, Kim KH, Kim YK, Nam SH, et al. Incidence of venous thromboembolism following major surgery in Korea: from the Health Insurance Review and Assessment Service database. J Thromb Haemost 2014;12:1035-43.

17. Kim DS, Park KM, Won YS, Kim JY, Lee JK, Kim JG, et al. Occurrence and prognosis of symptomatic venous thromboembolism in colorectal cancer surgery patients. Vasc Specialist Int 2014;30:4955.

18. Choi S, Lee KW, Bang SM, Kim S, Lee JO, Kim YJ, et al. Different characteristics and prognostic impact of deep-vein thrombosis/ pulmonary embolism and intraabdominal venous thrombosis in colorectal cancer patients. Thromb Haemost 2011;106:1084-94.

19. Tresoldi S, Flor N, Luciani A, Lombardi MA, Colombo B, Cornalba G. Contrast enhanced chest-MDCT in oncologic patients. Prospective evaluation of the prevalence of incidental pulmonary embolism and added value of thin reconstructions. Eur Radiol 2015; 25:3200-6.

20. Ageno W, Becattini C, Brighton T, Selby R, Kamphuisen PW. Cardiovascular risk factors and venous thromboembolism: a metaanalysis. Circulation 2008;117:93-102. 